\title{
Bilateral Orbital Peripheral Primitive Neuroectodermal Tumor: A Case Report
}

\author{
Ahmad Razif Omar ${ }^{1 *}$, Khairy Shamel Sonny Teo ${ }^{1}$, Ariffin Nasir ${ }^{2}$, Nur Asyilla Che Jalil ${ }^{3}$, Chandran Nadarajan ${ }^{4}$ and \\ Mohtar Ibrahim ${ }^{1}$ \\ ${ }^{1}$ Department of Ophthalmology, Universiti Sains Malaysia, Malaysia \\ ${ }^{2}$ Department of Paediatric, Universiti Sains Malaysia, Malaysia \\ ${ }^{3}$ Department of Pathology, Universiti Sains Malaysia, Malaysia \\ ${ }^{4}$ Department of Radiology, Universiti Sains Malaysia, Malaysia \\ Submission: July 13, 2018; Published: August 08, 2018 \\ *Corresponding author: Ahmad Razif Omar, Department of Ophthalmology, School of Medical Sciences, Health Campus, Universiti Sains Malaysia, \\ 16150 Kubang Kerian, Kelantan, Malaysia, Tel: +6019-4770779; Fax: +609-767 3370; Email: rayzief@gmail.com
}

\begin{abstract}
Peripheral primitive neuroectodermal tumors (pPNETs) are a group of small round-cell, neuroectodermal origin soft tissue tumors that arises outside the central or sympathetic nervous system. The definite diagnosis of pPNET is based on immunohistochemical examination. pPNET arising from the orbital region is very rare. To date, only unilateral cases of orbital pPNET have been reported. Herein, we present a unique case of bilateral orbital pPNET in 2-year old boy as an additional report to this rare disease.
\end{abstract}

Keywords: Peripheral primitive neuroectodermal tumour; Orbital pPNET; Childhood proptosis

\section{Introduction}

Primitive neuroectodermal tumor(PNET)isahighlymalignant tumor arising from cells that are believed to be retained from fetal brain development [1]. Therefore, most PNETs occur in the central nervous system (CNS). However, PNETs that occur outside of CNS are recognized as peripheral primitive neuroectodermal tumors [1,2]. Imaging and histopathological examination (HPE) such as light microscopy, immunohistochemistry (IHC) and cytogenetic features are helpful in recognizing pPNET $[3,4]$. Histologically, PNET is a small round blue cells tumor with highly cellular pattern, hyperchromatic nuclei and scanty cytoplasm $[1,4,5]$. IHC analyses has been helpful in distinguishing PNETs Case Report from other small, round, poorly differentiated tumors, such as rhabdomyosarcoma, neuroblastoma, and lymphoma $[2,4,5]$. pPNET has no gender predilection. It can occur in any age group but most commonly affects adolescents and young adults $[1,3,6]$. To the best of our knowledge, so far only 19 cases of orbital pPNET have been reported in literature worldwide. All the 19 reported cases have been unilateral presentation of the disease [1,7-10]. Apart from extremely rare disease, our case is unique because the pPNET arises from bilateral orbit with involvement of extradural site in the bilateral fronto-parietal region and extra-axial calvarial soft tissue.
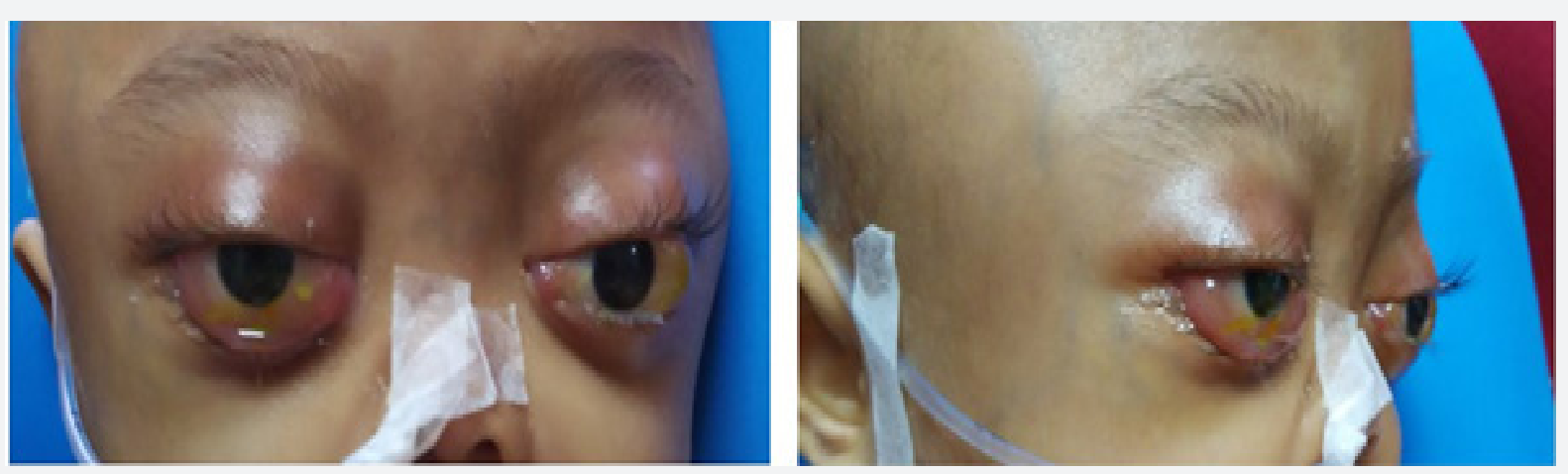

Figure 1: The pictures showing patient with severe bilateral proptosis, lagophthalmos, chemotic and prolapse conjunctiva before the chemotherapy. 
A previously healthy 2-year-old boy was admitted for 3-week history of persistent high-grade fever, poor oral intake and lethargy. He also had bilateral lid swelling and redness. There was no other ocular symptom, chills, rigors, limb weakness and seizures. Systemic review was unremarkable. Initial eye assessment showed bilateral swollen and erythematous eyelids. Extraocular muscle movement was normal with no eye deviations, proptosis or ptosis.

Within a week of hospitalisation, he rapidly developed severe proptosis in both eyes with lagophthalmos, conjunctival chemosis and prolapse (Figure 1). Extraocular muscle movement was almost completely restricted at this time. RAPD was equivocal and both pupils were mid-dilated with sluggish pupillary light response. There was presence of exposure keratopathy in both eyes and intraocular pressures were above $30 \mathrm{mmHg}$ bilaterally. Posterior segment examination bilaterally revealed choroidal folds and hyperaemic discs. Prompt treatment was initiated to reduce the IOP and control the exposure keratopathy.

Urgent magnetic resonance imaging (MRI) of brain and orbit was performed (Figure 2). The MRI showed multiple lobulated soft tissue masses in both orbits causing displacement of the extraocular muscles and optic nerves. The lesions caused expansion of the orbital cavity, significant periorbital swelling and marked proptosis. It was also associated with bifrontoparietal extradural collection, multifocal lobulated extra-axial calvarial soft tissue lesions as well as erosions of the orbital and calvarial bones adjacent to the masses.

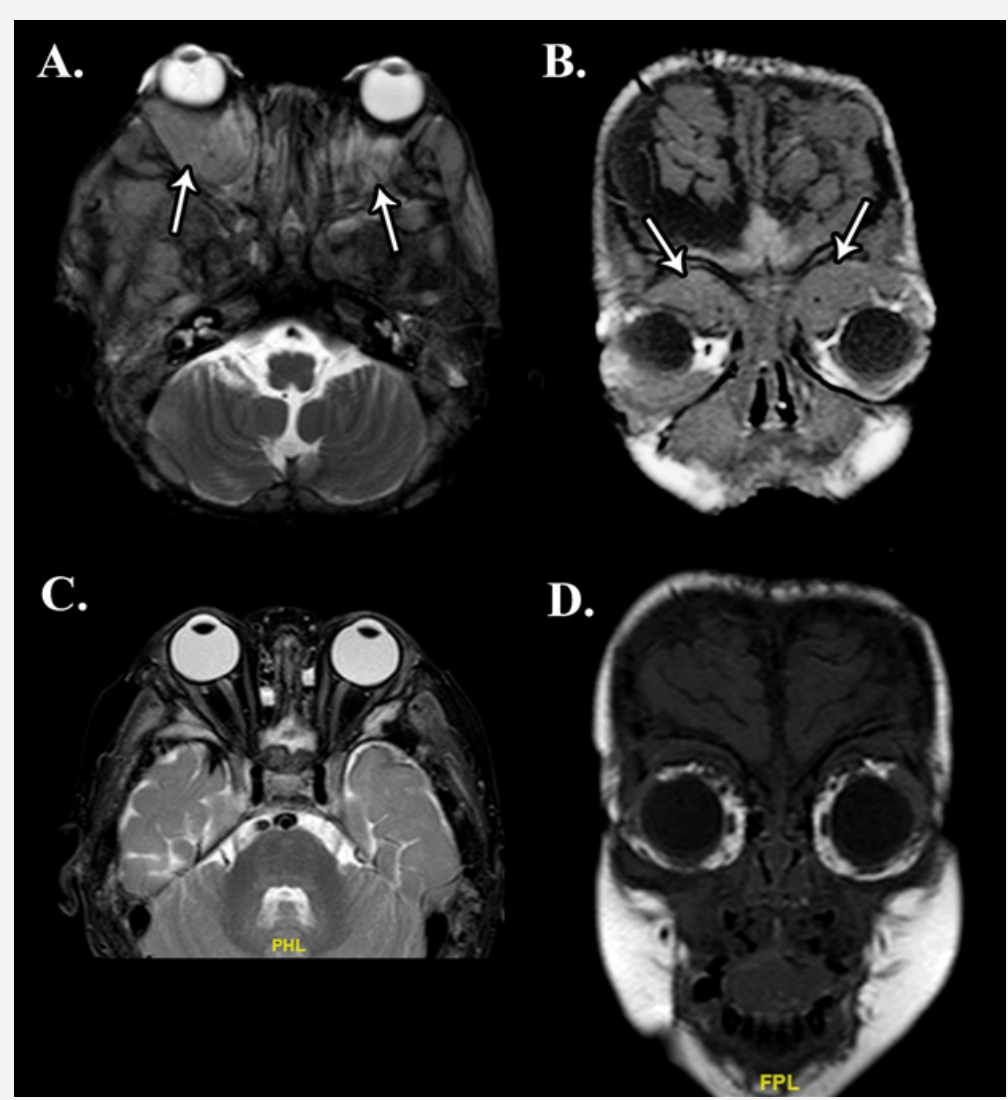

Figure 2A and 2B: Axial view of T2 weighted images of MR orbit and coronal view of T1 weighted images of MR orbit, show bilateral lobulated orbital mass with large right lateral extraconal mass extending to the retroorbital intraconal region causing displacement of the orbits. Note the lesions with similar appearance at bitemporal and posterior fossa region.

Figure 2C and 2D: Post-chemotherapy, axial view of T2 weighted images of MR orbit and coronal view of T1 weighted images of MR orbit, show previously seen soft tissue masses arising from all walls of bilateral orbits are no longer present.

He underwent urgent bilateral craniotomy and orbitotomy for tissue biopsy. Intraoperatively, there was extradural hemorrhagic fluid in multiple pockets, extensive tissue granulation with sclerosis, caseous material and erosion of the overlying bone. Subdural region and the brain parenchymal were found to be normal. HPE results from a tissue biopsy demonstrated the tumour cells as undifferentiated small round blue cells arranged in diffuse pattern. The cells had oval to round, deeply basophilic nuclei and inconspicuous nucleoli. The cytoplasm was scanty and indistinct with nuclear molding. Mitoses were abundant and clearly seen. The cells were positive for Synaptophysin, Chromogranin, CD99 and negative for Leucocyte Common Antigen (LCA), Desmin, CKAE1\&AE3 and GFAP. The diagnosis of pPNET was made based on these IHC findings and cytogenic analysis (Figure 3). 


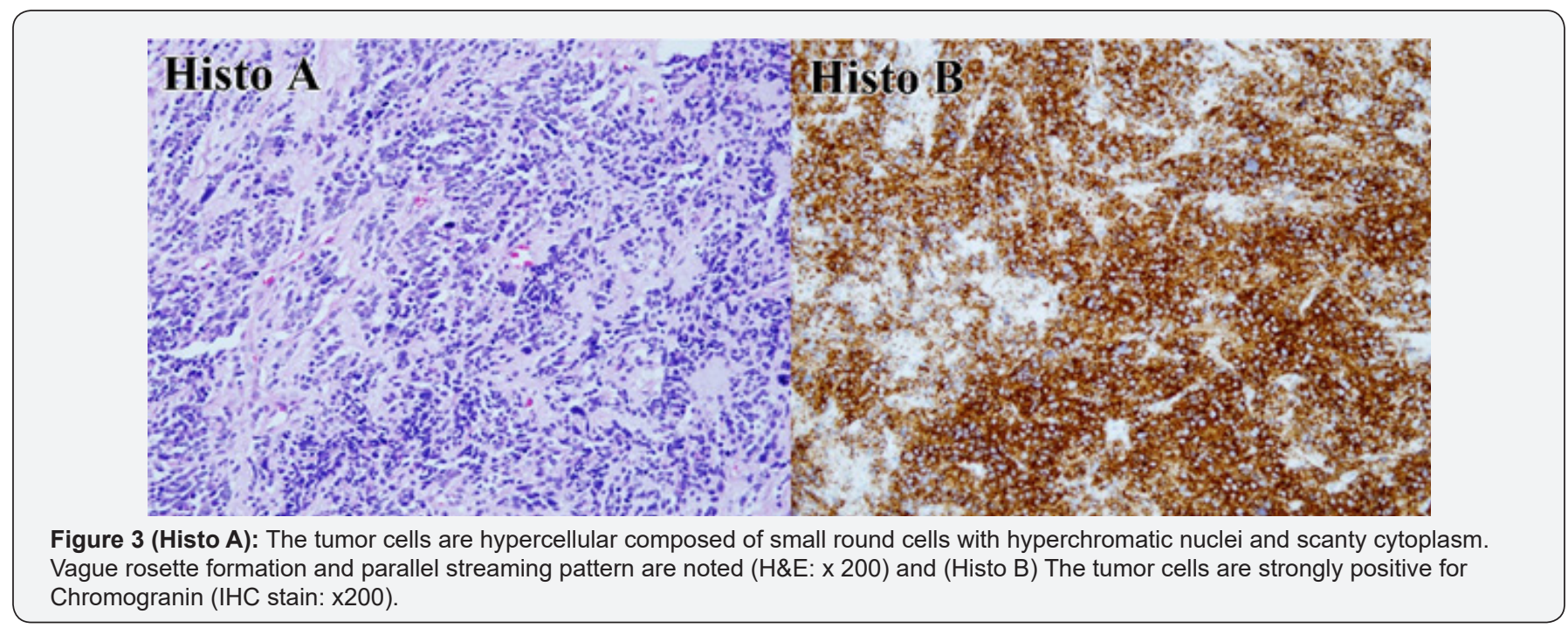

He was treated using modified Memorial Sloan Kettering Cancer Centre (MSKCC) chemotherapy protocol for PNET consisting of Cyclophosphamide, Adriamycin and Vincristine and Ifosphamide and Etoposide. There was significant clinical improvement after 3 cycles of chemotherapy. Exposure keratopathy resolved and IOPs were normal. Repeated radiological studies showed good response to chemotherapy. Bilateral intraorbital masses were no longer visualized and their contents showed normal signal intensities with no proptosis (Figure 2). He received another 3 cycles of chemotherapy and went into remission after the 6 cycles were completed. He was followed up regularly for another 10 months during which time there was no evidence of recurrence clinically and radiographically. Unfortunately, he defaulted subsequent imaging and oncology appointments due to social and logistic reasons. He succumbed to his illness as a result of intracranial extension and lung metastasis 5 months after his last appointment.

\section{Discussion}

Primitive neuroectodermal tumors (PNETs) are a highly malignant tumors arising from neuroepithelial stem cell that have not differentiated in a similar manner as a normal neuron and they appear as primitive cell. PNETs are believed to be retained from fetal brain development [1,7]. It is classified into two types based on its location; PNET and pPNET. Most PNETs occur in the brain and central nervous system (CNS) due to its embryological origin. However, PNETs that occur outside of CNS are recognized as peripheral primitive neuroectodermal tumors (pPNET) $[12,7,8]$.

PNETs usually affect adolescents and young adults and represent approximately $4 \%$ of all pediatric soft tissue tumors and have no sex predilection $[1,2,5]$. An orbital pPNETs tend to occur in younger patients when compared with other pPNETs. Only 4 out of the 19 published cases were older than 13 years at the time of diagnosis $[1,7,8,10]$. To the best of the authors' knowledge there were only 19 cases of orbital pPNET reported in the literature to date. None of the previous reported cases had bilateral eye involvement.

We report the only case of bilateral orbital pPNET with an extensive involvement of adjacent sites including extradural site in bifronto-parietal region and extra-axial calvarial soft tissue because of the highly metastatic feature and rapid growth of the pPNET tissue. There was also evidence of erosions in the orbital and calvarial bones adjacent to the soft tissue masses on radiological imaging. Besides that, an expansion of intraorbital pPNET mass retro-orbitally leads to displacement or proptosis of the globe $[6,11]$.

Histopathologically, PNET demonstrates small round cells which have a highly cellular pattern with hyperchromatic nuclei and scanty cytoplasm [1,3-5]. PNET is also capable of demonstrating varying grades of neural differentiation. HomerWright rosettes can be seen in certain cases [3]. Differential diagnoses of small round blue cells tumor in children include lymphoma, rhabdomyosarcoma, extra-adrenal neuroblastoma and metastatic nephroblastoma [6]. Immunohistochemical and cytogenetic studies help in the diagnosis confirmation of PNET. In this case, the diagnosis of pPNET was establised in view of IHC staining showed positivity towards synaptophysin, chromogranin, CD99 and negativity towards LCA, Desmin, S100 and GFAP $[1,3-5,12]$. PNET staging is done by radiological imaging of the brain and spinal column as well as spinal fluid microscopic examination.

Therapeutic strategy in PNET could be surgery, chemotherapy, chemoradiotherapy or combination $[1,3,5]$. The modality of treatment depends on the staging, type, site and size of tumour, extent of metastasis as well as the age and general health status of the patient $[5,8]$. Surgery was performed as the initial treatment for orbital pPNET in most reported cases $[1,5,8]$. However, complete surgical removal of the tumor is difficult. Treatment option for young children is chemotherapy alone, as radiation may interfere with their development [5]. The patient 
was treated well with MSKCC chemotherapy protocol for PNET using Cyclophosphamide, Adriamycin, Vincristine, Ifosfamide, Etoposide regime. This chemotherapy protocol had been used in latest orbital pPNET cases published and showed a good result $[1,5]$.

\section{Conclusion}

Orbital pPNET is extremely rare entity and this is the only case of bilateral orbital pPNET to have been reported thus far in medical literature [1,7-9]. Clinical diagnosis in this case was challenging due to vague presentation. The definitive diagnosis was reached with the IHC studies supported by the modalities of imaging and HPE [5]. Chemotherapy modality is considered as the best treatment option with the good result in this case.

\section{Acknowledgements}

The author reports no conflict of interest in this work.

\section{Consent}

Written informed consent was obtained from the patient for publication of this case report and any accompanying images. A copy of the written consent is available for review by the Editorin-Chief of this journal.

\section{References}

1. Romero R, Castano A, Abelairas J, Peralta J, Garcia-Cabezas MA, et al (2011) Peripheral primitive neuroectodermal tumour of the orbit. Br J Ophthalmol 95(7): 915-920.

2. Dehner LP (1986) Peripheral and central primitive neuroectodermal tumors: A nosologic concept seeking consensus. Arch Pathol Lab Med 110(11): 997-1005.

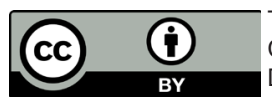

This work is licensed under Creative Commons Attribution 4.0 License DOI: $10.19080 /$ JOJO.2018.06.555700
3. Jones JE, McGill T (1995) Peripheral primitive neuroectodermal tumors of the head and neck. Arch Otolaryngol Head Neck Surg 121(12): 13921395.

4. Alyahya GA, Heegaard S, Fledelius HC, Rechnitzer C, Prause JU (2000) Primitive neuroectodermal tumor of the orbit in a 5-year-old girl with microphthalmia. Graefe's Arch Clin Exp Ophthalmol 238(9): 801-806.

5. Kim UR, Arora V, Devanand J, Khazei HM (2009) Multimodality treatment approach in management of primary peripheral primitive neuroectodermal tumor of the orbit. Indian J Ophthalmol 57(5): 395398.

6. Bansal RK, Gupta A (1995) Primitive neuroectodermal tumour of the orbit: A case report. Indian J Ophthalmol 43: 29-31.

7. G Santra, P K Sinha, D De, S Phaujdar (2011) Peripheral type of primitive neuroectodermal tumour arising from the left orbital floor. Singapore Med J 52(6): e138-e140.

8. Hacer Bal, Cennet Şahin, Erkin Aribal (2013) An extremely rare case of a pediatric peripheral primitive neuroectodermal tumour: Orbital primitive neuroectodermal tumour. Marmara Medical Journal 26: 171173.

9. Chen HM, Chuang WY, Chu HY, Tan HY (2014) Localized peripheral primitive neuroectodermal tumor of the conjunctiva: a rare presentation. Eye 28(9): 1143-1149.

10. Chokthaweesak W, Annunziata CC, Alsheikh O, Ng JD, Wilson DJ, et al. (2011) Primitive neuroectodermal tumor of the orbit in adults: a case series. Ophthal Plast Reconstr Surg 27(3): 173-179.

11. Singh AD, Husson M, Shields CL, et al. (1994) Primitive neuroectodermal tumour of the orbit. Arch Ophthalmol 112: 217-221.

12. Das D, Kuri GC, Deka P, Bhattacharjee K, Bhattacharjee H, Deka AC (2009) Primary primitive neuroectodermal tumor of the orbit. Indian J Ophthalmol 57(5): 391-393.

\section{Your next submission with Juniper Publishers will reach you the below assets}

- Quality Editorial service

- Swift Peer Review

- Reprints availability

- E-prints Service

- Manuscript Podcast for convenient understanding

- Global attainment for your research

- Manuscript accessibility in different formats

( Pdf, E-pub, Full Text, Audio)

- Unceasing customer service

Track the below URL for one-step submission https://juniperpublishers.com/online-submission.php 